Int. J. Dev. Biol. 58: 635-642 (2014)

doi: $10.1387 / \mathrm{ijdb} .140116 \mathrm{df}$

\title{
The dynamics of alkaline phosphatase activity during operculum regeneration in the polychaete Pomatoceros lamarckii
}

\author{
RÉKA SZABÓ and DAVID E.K. FERRIER* \\ The Scottish Oceans Institute, Gatty Marine Laboratory, University of St Andrews, Fife, UK
}

\begin{abstract}
Alkaline phosphatase enzymes are found throughout the living world and fulfil a variety of functions. They have been linked to regeneration, stem cells and biomineralisation in a range of animals. Here we describe the pattern of alkaline phosphatase activity in a spiralian appendage, the operculum of the serpulid polychaete Pomatoceros lamarckii. The P. lamarckii operculum is reinforced by a calcified opercular plate and is capable of rapid regeneration, making it an ideal model system to study these key processes in annelids. Alkaline phosphatase activity is present in mesodermal tissues of both intact and regenerating opercular filaments, in a strongly regionalised pattern correlated with major morphological features. Based on the lack of epidermal activity and the broad distribution of staining in mesodermal tissues, calcification- or stem cell-specific roles are unlikely. Transcriptomic data reveal that at least four distinct genes contribute to the detected activity. Opercular alkaline phosphatase activity is sensitive to levamisole. Phylogenetic analysis of metazoan alkaline phosphatases indicates homology of the $P$. lamarckii sequences to other annelid alkaline phosphatases, and shows that metazoan alkaline phosphatase evolution was characterised by extensive lineage-specific duplications.
\end{abstract}

KEY WORDS: serpulid, calcification, biomineralisation, appendage regeneration

\section{Introduction}

Alkaline phosphatases (ALPs, EC 3.1.3.1) are metalloenzymes found throughout the living world and involved in a wide variety of biological processes including metabolite processing, transport and secretion, stem cell function and biomineralisation. In mammals, ALP proteins are typically membrane-bound via a GPI anchor (Buchet et al., 2013), but they can also be shed from the plasma membrane into extracellular spaces including the gut lumen. Likewise, the intestinal alkaline phosphatases of the silkworm include both membrane-bound forms and a soluble isozyme present in the gut lumen (Eguchi, 1995). Most metazoan species possess multiple alkaline phosphatase genes. There are few phylogenetic studies of metazoan alkaline phosphatases, but existing data indicate frequent lineage-specific duplications both within specific clades of vertebrates and more broadly among bilaterian groups (Yang et al., 2012).

Mammalian alkaline phosphatases are active in a variety of organs involved in secretion or absorption of materials such as the kidney, lung and intestine. Invertebrate alkaline phosphatases appear to have a similar association with transport: ALP activity has been reported in excretory organs of insects and annelids, salivary glands of insect larvae, and the guts of diverse species (Myohara, 2004; Eguchi, 1995; Hasse et al., 2010; Kitamura and Shimizu, 2000).

Alkaline phosphatases are considered stem cell markers, and may fulfil roles in regeneration in some animals. ALP activity is a sensitive marker of pluripotency in human embryonic stem cells (O'Connor et al., 2008) and is present in putative stem cells of ascidians (Rinkevich etal., 2013), parasitic crustaceans (Shukalyuk et al., 2005) and hydrozoans (Isaeva et al., 2011). ALPs are also active in the mammalian germ line (Buchet et al., 2013). Alkaline phosphatase activity is increased in the coelomic epithelium of regenerating arms in a sea star (Donachy et al., 1990). During amphibian limb regeneration ALP activity was detected in multiple cell types, including degenerating muscle fragments and blastemal cells (Schmidt and Weary, 1962). ALP activity was also reported from planarian neoblasts (Osborne and Miller, 1963). However, in

Abbreviations used in this paper: ALP, alkaline phosphatase.

\footnotetext{
*Address correspondence to: David E.K. Ferrier. The Scottish Oceans Institute, Gatty Marine Laboratory, University of St Andrews, East Sands, St Andrews, Fife, KY16 8LB, UK. Fax: +44-(0)1334-463443. Tel.: +44-(0)1334-463480. e-mail: dekf@ st-andrews.ac.uk - web: http://synergy.st-andrews.ac.uk/edge
}

Accepted: 20 June 2014.

ISSN: Online 1696-3547, Print 0214-6282 
the highly regenerative annelid Enchytraeus japonensis, Myohara (2004) failed to detect any ALP activity in regeneration blastemas, neoblasts or gonads.

In vertebrates, alkaline phosphatases have a well-established role in tissue mineralisation. In humans and mice, deficiency of Tissue Non-specific Alkaline Phosphatase or TNAP (hypophosphatasia) causes a bone mineralisation defect of varying severity (Millán, 2013). Alkaline phosphatase activity potentially associated with mineralisation has also been reported in a variety of invertebrate systems, including the mantle epithelia and larval shell fields and shell glands of molluscs (Timmermans, 1969; Hohagen and Jackson, 2013), in calcifying cell cultures of corals (Domart-Coulon et al., 2001), and in the calcifying integument of crustaceans (Chockalingam, 1971), although almost nothing is known about the functional relevance of phosphatase activity to mineralisation in these systems.

The serpulid polychaete $P$. lamarckii is a sessile, tube-dwelling filter feeder of rocky intertidal zones. Like most serpulids, it possesses a head appendage, the operculum, which is an evolutionary novelty of this polychaete family. Opercula are defensive tube plugs and can be autotomised to escape predation or mechanical damage. In Pomatoceros and related genera, the organ is reinforced by a calcareous opercular plate, making it one of the rare instances of mineralised hard parts in annelids (Bubel et al., 1983). Upon autotomy, the $P$. lamarckii operculum and its peduncle (together making up the opercular filament) regenerate rapidly (Bubel et al., 1985; Szabó and Ferrier, 2014); making this unique appendage an excellent system in which to study both appendage regeneration and calcification from the perspective of a spiralian animal.

Here, we provide a description of alkaline phosphatase activity during opercular regeneration in order to explore the potential role of these enzymes in annelid appendage regeneration and calcification. The P. lamarckii opercular filament displays strong, patterned alkaline phosphatase activity in its mesodermal tissues.
This activity is inhibited by levamisole in a dose-dependent fashion. Proximodistally regionalised activity appears early in regeneration, and exhibits both persistent and dynamic features. The cup-shaped operculum displays little to no ALP activity in mature specimens but possesses regions of high activity in regenerates of all stages, hinting at a regeneration-specific role for these enzymes in the operculum. Appreciable staining is absent from the epidermis at all stages, including the calcifying opercular plate epidermis. Thus, alkaline phosphatases are unlikely to function in mineral deposition in this species. The absence of activity in the proliferating epidermis and the broad distribution and persistence of activity within mesodermal regions also argue against a stem cell-specific function. At least four distinct alkaline phosphatases are expressed in mature and regenerating opercular filaments. Phylogenetic analysis of these and other metazoan ALP protein sequences reveals a dynamic evolutionary history for this important family.

\section{Results}

\section{Anatomy of the opercular filament}

The $P$. lamarckii opercular filament consists of an operculum (cup) sitting atop a sturdy peduncle (Fig. 1A). Distally, the operculum ends in a calcified end plate bearing a central spine (Bubel et al., 1983). Cup and peduncle are lined with a thick columnar epidermis (Fig. 1, Fig. 2B). The rim of the opercular plate is characterised by particularly tall epidermal cells (Fig. 2D, arrowheads). The bulk of the cup is filled by connective tissue (Hanson, 1949; Fig. 2B, D), with a large spiral formed by the blind end of the opercular blood vessel occupying a central portion (Fig. 2B). The blood vessel runs the length of the peduncle in a straight line. Within the peduncle, a thick bundle of muscle fibres is situated ventrally, while connective tissue is concentrated on the dorsal side (Hanson, 1949). The cup and the peduncle are separated by a distinct groove, and the distal end of the peduncle bears a pair of lateral "wings". The

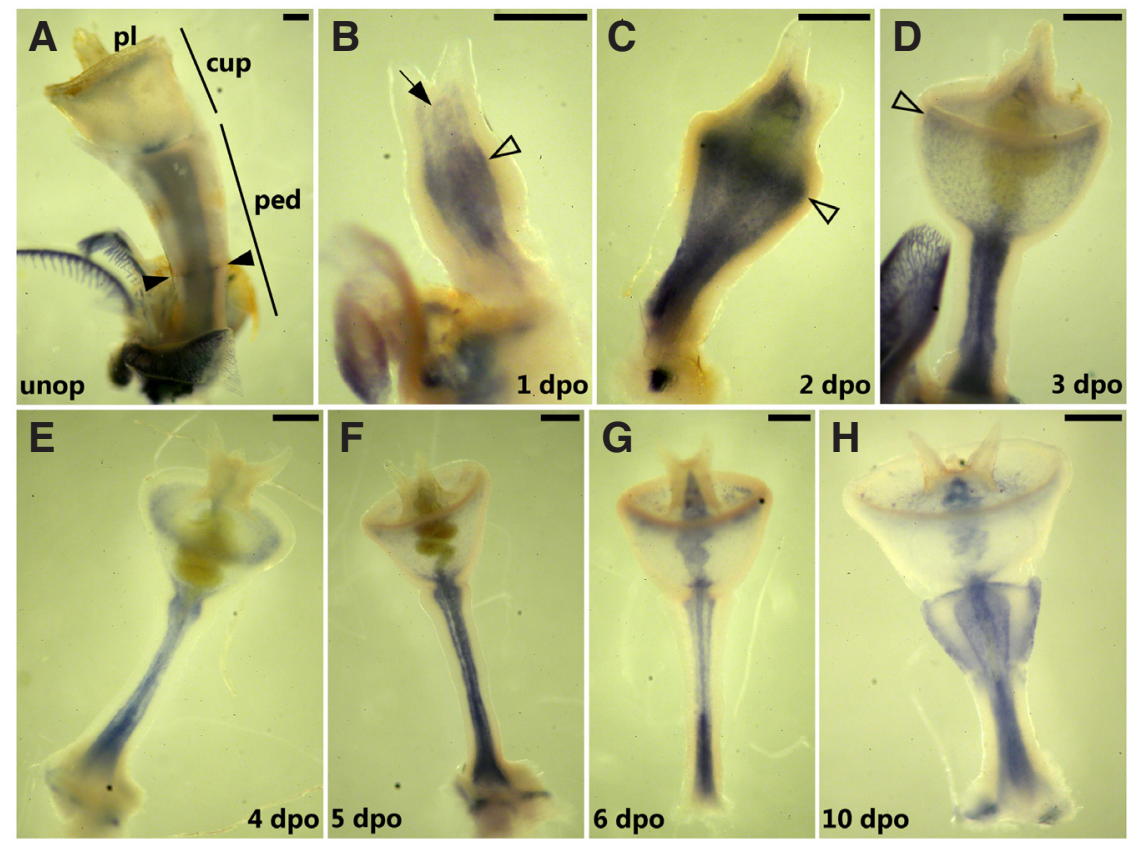

Fig. 1. General pattern of alkaline phosphatase activity in intact and regenerating opercula of $P$. lamarckii. (A) Mature opercular filament with major regions labelled (ped, peduncle; pl, plate). Arrowheads indicate the autotomy plane known as the easy break point (ebp), the site of all amputations in this study. Note absence of cup staining. The dark band under the opercular plate is probably endogenous pigmentation. (B) Young regenerate 1 day post-operation (dpo). Note the more strongly stained swelling region, and the outline of the opercular blood vessel, highlighted distally by the arrow. Empty arrowheads in $(B, C$ and $D)$ highlight the development of the distal ring. (C) 2 dpo regenerate undergoing cup morphogenesis. The distal ring of darker staining is evident. The opercular blood vessel inside the presumptive cup has expanded into a large spiral, here filled with greenish haemolymph. The strongly coloured haemolymph can obscure staining in the blood vessel wall. (D) 3 dpo. The distal ring is now restricted to a narrow region just under the rim. (E) 4 dpo regenerate with a differentiated cup and peduncle. A clear proximodistal difference in staining intensity in the peduncle is generally absent at this time. (F) 5 dpo regenerate. Some specimens show stronger staining in the proximal than in the distal peduncle by this point. (G) 6 dpo regenerate with a clear mid-peduncle boundary in staining localisation. Note the strongly stained blood vessel, base of the cup and distal ring. (H) 10 dpo regenerate. Extensive, non-specific dye precipitation has occurred on the cuticle of the distal peduncle, but does not obscure the underlying specific mesodermal staining. All views are dorsal, with distal end upper-most. Scale bars are approximately $0.2 \mathrm{~mm}$. 

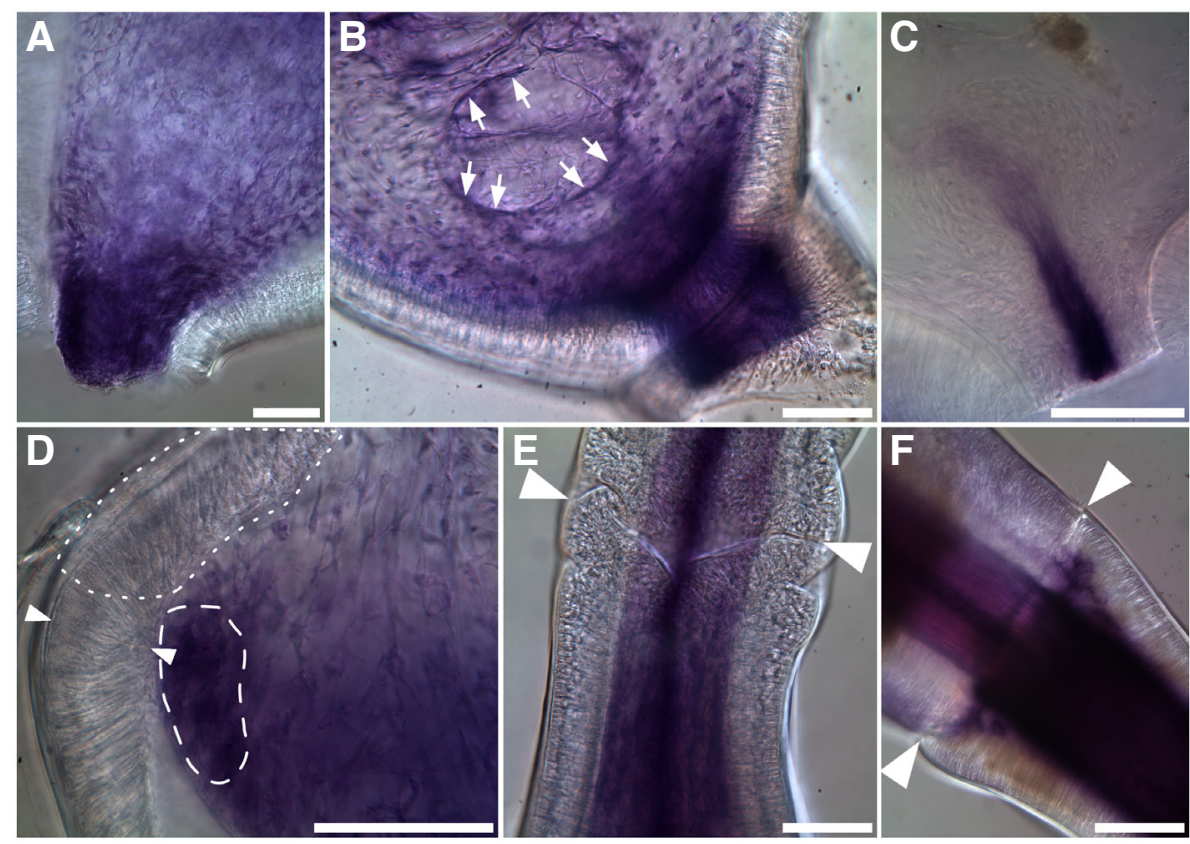

Fig. 2. Notable features of opercular alkaline phosphatase staining. (A-C) Development of connective tissue staining in the proximal portion of the cup. Half cups cut along the plane of left-right symmetry. (A) A gradient in staining intensity in the mesenchyme from base to interior of the cup is already apparent at $3 \mathrm{dpo}$. The blood vessel is out of focus in this image. (B) 6 dpo half cup showing a well-developed proximodistal gradient. The walls of the blood vessel, seen in the central area of the cup, are heavily stained (small arrows). (C) Base of an unoperated cup. Only the proximal portion of the blood vessel is stained; connective tissue is negative throughout the cup. (D) Rim region of a 3 dpo cup. The dark distal ring (dashed line) is apparent adjacent to the thickened epithelium of the rim (small arrowheads). The opercular plate epidermis is marked by a dotted outline. Note the complete lack of epidermal staining, particularly in the opercular plate, which is calcifying at this stage. (E) Mid-peduncle region of a 5 dpo regenerate showing weak proximodistal regionalisation in staining intensity. (F) Mid-peduncle region of a mature opercular filament displaying strong regionalisation. Arrowheads in (E,F) mark the location of the easy break point. Distal is towards the top in all images. Scale bars are $100 \mu$ m.

peduncle itself is divided into two roughly equal parts by the easy break point (Fig. 1A, 2F); this is the plane of autotomy, and it is the location of all experimental amputations in this study. Distal to the easy break point, the mature peduncle has a pronounced triangular cross section (Fig. $3 \mathrm{C}, \mathrm{D}$ ), whereas the proximal half is much more rounded (Fig. 3B).

After amputation, the operculum regenerates rapidly. The stump, consisting of the part of the peduncle proximal to the easy break point, usually develops a swelling within 1-2 days (Fig. 1B). This swelling develops into the future cup. From 2-3 days post-operation (dpo) onwards, the regenerate elongates and differentiates into a cup and peduncle (Fig. 1 C-D), eventually reacquiring anatomical features of the mature opercular filament such as the distal lateral wings of the peduncle, the operculo-peduncular groove and the easy break point (Fig. $1 \mathrm{E}-\mathrm{H}$ ). By about a week after amputation, the regenerate generally possesses all major features of the mature opercular filament, although further growth and the development of pigmentation can take several weeks. Beginning around 2-3 $\mathrm{dpo}$, the opercular plate epidermis secretes calcareous material mainly composed of aragonite (Bubel et al., 1983; 1985; Szabó et al., 2014).

\section{Alkaline phosphatase activity in the opercular filament}

Both mature and regenerating opercular filaments display strong and regionalised alkaline phosphatase activity (Fig. $1 \mathrm{~A}-\mathrm{H})$. At all stages, staining is absent from the epidermis but present in the mesodermal tissues of the peduncle, as well as the walls of the peduncular portion of the opercular blood vessel. There is evidence for proximodistal regionalisation in the staining pattern, and different aspects of this pattern develop at different times. Already at swelling stage (1-2 dpo), a ring of stronger staining can often be observed around the distal margin of the swelling, with most of the presumptive cup region being lighter (Fig. $1 \mathrm{~B}, \mathrm{C}$ ). As an operculum and a peduncle begin to differentiate and expand, this pattern becomes more pronounced (Fig. 1D). During cup differentiation, the ring is restricted to the distal-most part of the cup, just below the rim, and the contrast in staining intensity between the ring and the remainder of the cup is enhanced (Fig. $1 \mathrm{D}-\mathrm{E}$ ). As the cup and groove differentiate, strong staining at the base of the cup also becomes apparent (Fig. $1 \mathrm{D}-\mathrm{H})$. The distal ring and the basal region of strong staining persist late into regeneration (Fig. $1 \mathrm{H})$, although they are not typically present in unoperated opercula (Fig. 2C).

Typically around 3-4 dpo, a groove also begins to form between the peduncle and cup; the constriction of mesenchyme in this region is accompanied by pronounced ALP staining that fades out more distally inside the cup (Fig. 1D; Fig. 2 A-B). Throughout regeneration, the spiral opercular blood vessel is typically stained (Fig. 1 B,G,H; Fig. 2B), although individual variation occurs and fainter staining can be difficult to see if the vessel is still filled with haemolymph. In mature opercula, the cup connective tissue and blood vessel are often unstained, although blood vessel staining is maintained in the peduncle (Fig. 2C).

From around 5-6 dpo, regionalisation of staining becomes evident within the peduncle. Commonly, the proximal half of the peduncle appears more strongly and uniformly stained than the distal half (Fig. 1 F-H, Fig. 2F). The boundary in staining intensity is not necessarily sharp, and it is situated close to the easy break point, which is usually apparent by $6 \mathrm{dpo}$ (Fig. 2 E,F). This midpeduncle boundary is maintained in mature peduncles (Fig. 3A shows a particularly strong example).

To examine the distribution of staining within the peduncle, a few fixed specimens from each stage were cut into blocks. In late regenerating and mature peduncles, transverse cuts show a different distribution of staining between proximal and distal peduncles. Proximally, where the peduncle has a more rounded cross section, staining appears in the blood vessel wall and in a broad concentric ring immediately beneath the epidermis (Fig. 3B). 

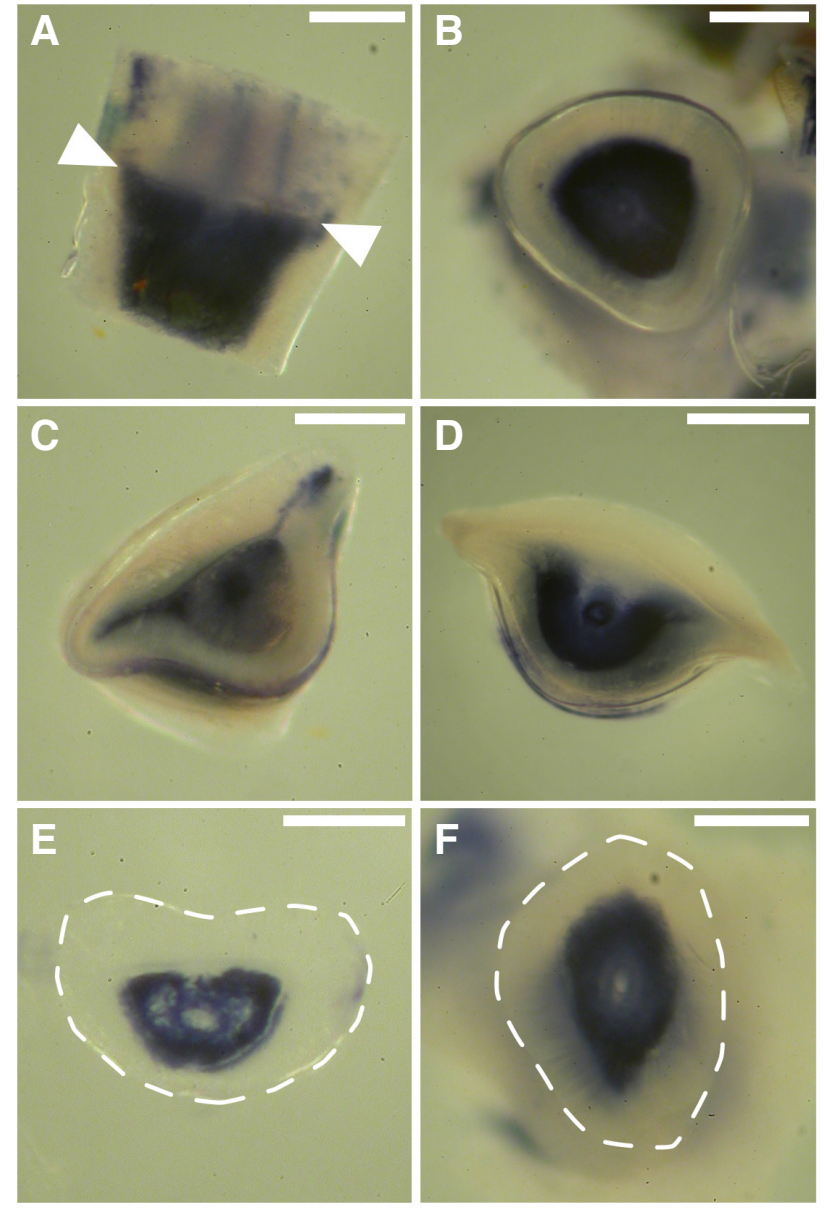

Distal peduncles of late regenerates and mature specimens appear to display two different patterns. Some possess strong staining only in the dorsal corners of the triangular cross-section of the peduncle (3/9 mature peduncles, Fig. $3 \mathrm{C}$ ), whereas in others, the strongest staining forms a horseshoe shape with a gap along the dorsal side of the mesodermal tissues (6/9 mature peduncles, Fig. 3D). Early regenerates only display a concentric pattern (Fig. 3F).

Levamisole inhibits some but not all alkaline phosphatases. We tested whether the opercular ALPs of $P$. lamarckii are levamisolesensitive by performing staining experiments in the presence of the inhibitor. 6 dpo regenerates stained in the presence of $2 \mathrm{mM}$ or $10 \mathrm{mM}$ levamisole show reduced staining compared to controls
Fig. 3. Spatial and temporal variation in peduncle staining. (A) Dorsal view of middle third of a mature peduncle with a particularly clear difference between the proximal (bottom) and distal (top) regions. The boundary is at the easy break point, marked by arrowheads. (B) Distal view of the proximal third of a mature peduncle, showing the even distribution of staining within the mesoderm. The blood vessel occupies a central position. (C) Distal view of the partial peduncle shown in (A). This specimen displays the more restricted staining pattern seen in mature peduncles. The blood vessel in the middle is also marked with strong staining. Dorsal is uppermost in (C,D and E). Note purple precipitate in the ventral cuticle of $C$ and $D$ is non-specific staining. (D) Proximal view of a mature distal peduncle (cut at the same level as A) showing the stronger mature staining pattern. Strong staining is present in the blood vessel wall (dark ring in the centre) and in a broad horseshoe pattern excluding the dorsal part of the mesoderm. (E) 6 dpo distal peduncle, showing a cut surface equivalent to that in (D). Dashed line is the outline of the specimen. (F) Distal view of a 1 dpo regenerate cut just below the swelling. Staining is uniform around the circumference at this stage. As in (F), the boundary of the cut surface is outlined. Scale bars are approximately $0.1 \mathrm{~mm}$.

(Fig. 4). The reduction is more marked in the group subjected to the stronger treatment, consistent with dose-dependent inhibition of alkaline phosphatase activity.

\section{P. lamarckii alkaline phosphatase sequences and metazoan ALP evolution}

We used transcriptomic data generated from unoperated, 2 dpo and 6 dpo opercular filaments (unpublished data) to identify several distinct alkaline phosphatases in P. lamarckii. In addition to an alkaline phosphatase sequence from the larval transcriptome of Kenny and Shimeld (2012), three full-length alkaline phosphatase domains were successfully assembled from $100 \mathrm{bp}$ paired-end reads. Three reads corresponding to a conserved region of the alkaline phosphatase domain may represent another novel ALP. An alignment of the four predicted Pomatoceros alkaline phosphatase domains and the fragment with human placental ALP as a reference is shown in Fig. 5. The alkaline phosphatase domain, which makes up most of a typical alkaline phosphatase protein, is highly conserved across the metazoans included in this analysis (see Supplementary Information), and is notably absent from the currently available poriferan and ctenophore data.

Phylogenetic analysis of the ALP complements of diverse animals using distance and likelihood methods gives trees with many highly and consistently resolved branches near the tips, but low resolution at deeper nodes (Fig. 6). P. lamarckii sequences consistently group with those of the other annelid, Capitella teleta. Vertebrate
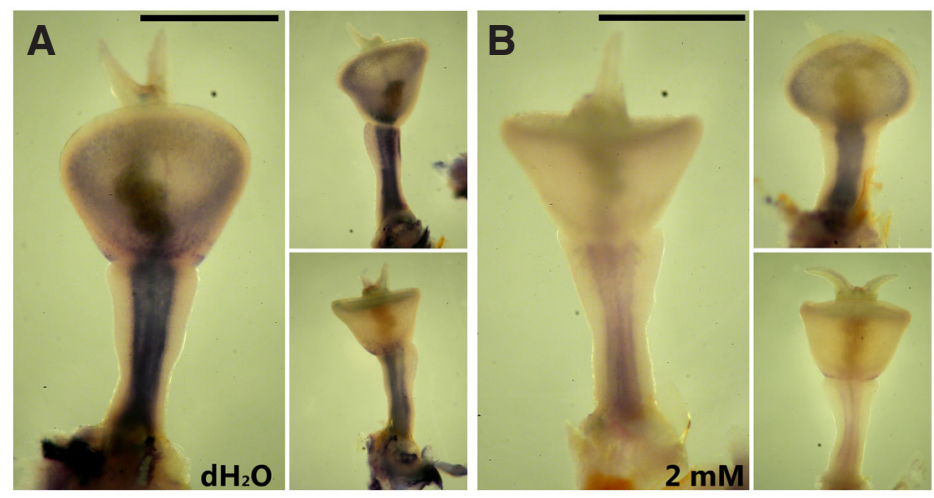
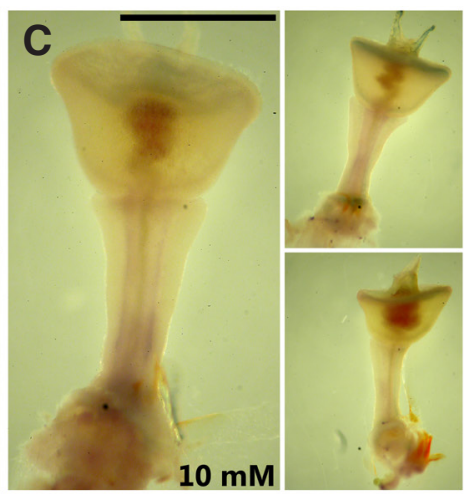

Fig. 4. Opercular alkaline phosphatases are levamisole sensitive. 6 dpo regenerates exposed to (A) $\mathrm{dH}_{2} \mathrm{O}$ control, (B) $2 \mathrm{mM}$, and (C) 10 $\mathrm{mM}$ levamisole treatments during staining for alkaline phosphatase activity. All specimens were stained for 50 minutes at room temperature. Small images next to each main panel illustrate the range of variation from most (top) to least (bottom) staining in each sample. Scale bars in the main images are approximately $0.5 \mathrm{~mm}$. 
ALP domains cluster in the two main clades ("intestinal/placental" and "tissue-nonspecific") expected from previous studies (Yang et al., 2012). In general, major lineages each appear to have distinct complements of alkaline phosphatase sequences, with no clear orthology across phyla.

\section{Discussion}

In both intact and regenerating opercular filaments of $P$. lamarckii, there is a striking contrast in alkaline phosphatase activity between the epidermis and other tissues. In the peduncle, strong mesodermal staining is always present, whereas the epidermis does not appear to possess significant activity in any part of the opercular filament. However, details of the mesodermal staining pattern change during regeneration. Most notably, the proximodistal differentiation of the peduncle and the formation of an autotomy plane are accompanied by a marked regionalisation in the distribution of ALP staining. In early regenerates and in more mature proximal peduncles, the strongest staining forms a uniform band just underneath the epidermis. In late regenerates and mature opercular filaments, the distal peduncle loses staining in some areas, resulting in a horseshoe shape or even a strongly reduced staining pattern concentrated in the dorsal corners of the peduncle.
Aside from the large blood vessel running proximodistally through the entire opercular filament, the mesodermal portion of $P$. lamarckii peduncles contains longitudinal muscles and connective tissue described as "cartilage" by Hanson (1949). The spatial and temporal variation in ALP activity in the peduncle may be explained by the changing distributions of ALP-positive tissues. Whether the two different staining patterns observed in mature distal peduncles, where strong staining may be limited to the blood vessel and two small corners or encompass most of the mesoderm (Fig. 3 C-D), are different phases of a dynamic pattern or simply individual variation, and what the significance of this variation might be, is at present unclear.

The fact that strong and widespread ALP activity persists in the peduncle and the blood vessel of intact opercular filaments is perhaps interesting in itself. In other annelids, alkaline phosphatases display fairly restricted activity both in space and in developmental time. Although Kitamura and Shimizu (2000) and Hasse etal., (2010) are studies of embryos and juveniles, Myohara's (2004) observations on adult $E$. japonensis worms suggest that widespread ALP activity in mesodermal tissues is, at the very least, not a universal feature of annelids. Interestingly, alkaline phosphatase activity in muscle tissue of a euryhaline crustacean appears to respond to changes in environmental salinity (Pinoni et al., 2005). A role in
physiological acclimation would be plausible in an intertidal animal like $P$. lamarckii, and the horseshoe pattern of staining in distal peduncles is consistent with activity in peduncular muscle or associated connective tissue.

Most of the P. lamarckii operculum itself (the cup) is filled with a cartilage-like connective tissue with large amounts of extracellular matrix (Hanson, 1949). In mature opercula, this tissue does not show appreciable ALP activity. In contrast, regenerating cups contain strongly stained cells near the base and in a distal ring, with weaker staining between them (Fig. 1 C-H; Fig. 2 A-D). In vertebrates, ALP activity is a characteristic of hypertrophic cartilage (Buchet et al., 2013), which is associated with ossification. However, the mineralised portion of the opercular plate in serpulids is secreted by the plate epidermis (Bubel et al., 1983). Thus, it seems more likely that the activity seen in

hs $a-a l p p$ pla-alp1 $p l a-a l p 2$ pla-alp3 pla-alp4 $p 1 a-r 2 b$

hsa-alpp $p l a-a l p 1$ pla-alp2 pla-alp3 pla-alp4 $p 1 a-r 2 b$

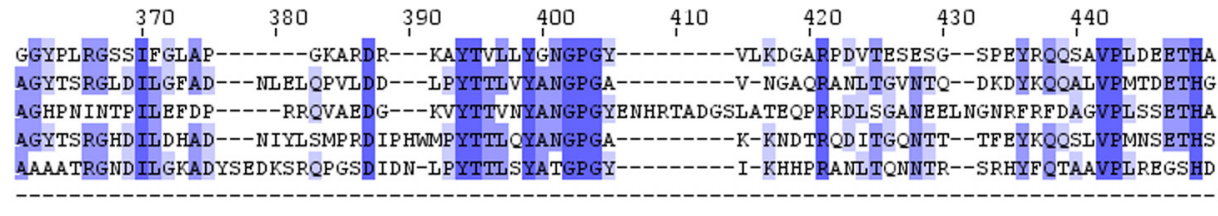

480

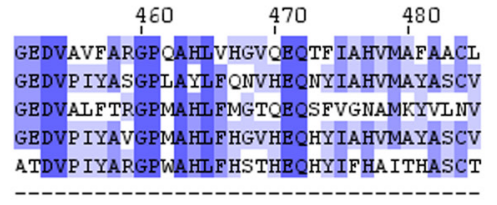

Fig. 5. Sequences of opercular alkaline phosphatases (ALP). Alignment of the four fulllength alkaline phosphatase domains and a small fragment of a possible fifth ALP predicted from transcriptomic data with human placental alkaline phosphatase (hsa-alpp) as a reference. Pla-alp1 is from Kenny and Shimeld (2012). The name of the fragment (pla-r2b) stands for conserved region 2, variant $b$. 
the regenerating cup has a different developmental role, perhaps in the secretion and/or maturation of the extracellular matrix that forms the bulk of the opercular connective tissue.

The apparent lack of staining in the epidermis of calcifying opercular plates is particularly noteworthy, as ALP activity has been linked to mineralisation in other animals. In vertebrates, while the exact role of tissue-nonspecific alkaline phosphatase (TNAP) in bone formation has been debated, the fact of its involvement is firmly established (Millán, 2013). One of the proposed functions of TNAP in bone formation is the regulation of inorganic pyrophosphate levels, as pyrophosphate is an inhibitor of calcification, a function that could theoretically operate in calcifying invertebrates that secrete calcium carbonate rather than calcium phosphate, the main component of bone mineral. Furthermore, although functional experiments in invertebrates are lacking, alkaline phosphatase activity has been reported from $\mathrm{CaCO}_{3}$ producing invertebrate systems including mollusc mantles (Timmermans, 1969) and cultured coral cells (Domart-Coulon et al., 2001). Thus, an involvement of ALPs in serpulid calcification has a prioriplausibility. However, given the lack of appreciable staining in calcifying opercular plates, our data do not support such a role in the $P$. lamarckii operculum.

Alkaline phosphatases have also been linked to stem cells in some organisms (O'Connor et al., 2008; Rinkevich et al., 2013; Shukalyuk et al., 2005; Isaeva et al., 2011). We currently do not know whether opercular regeneration in $P$. lamarckii involves a stem cell system. However, previous studies (Bubel et al., 1985) and our observations (Szabó and Ferrier, 2014) indicate that the epidermis is the most proliferative tissue during regeneration, whereas it showed the least alkaline phosphatase activity in the present study. In contrast, mesodermal ALP staining is strong in mature peduncles and much more general than expected from a stem cell marker. While the presence of ALP-positive stem cells in the regenerating opercular filament cannot be excluded at present, opercular alkaline phosphatases are clearly not restricted to stem cell-related roles.

We recovered four distinct alkaline phosphatase genes from transcriptomic datasets derived from unoperated and regenerating opercular filaments (unpublished data), consistent with our finding ALP activity in this appendage in this study. Although ALP activity as measured by a colour reaction is quite broad in both intact and regenerating opercula, it would be interesting to distinguish the expression domains of the four genes within those regions. In mammals, spatially distinct expression of several alkaline phosphatase genes characterises organs such as the small intestine and kidney (Buchet et al., 2013). It would be surprising if this were not the case for annelids like Pomatoceros. In addition to the four complete ALP domains, we also found a small fragment represented by only three reads, corresponding to a conserved motif within the

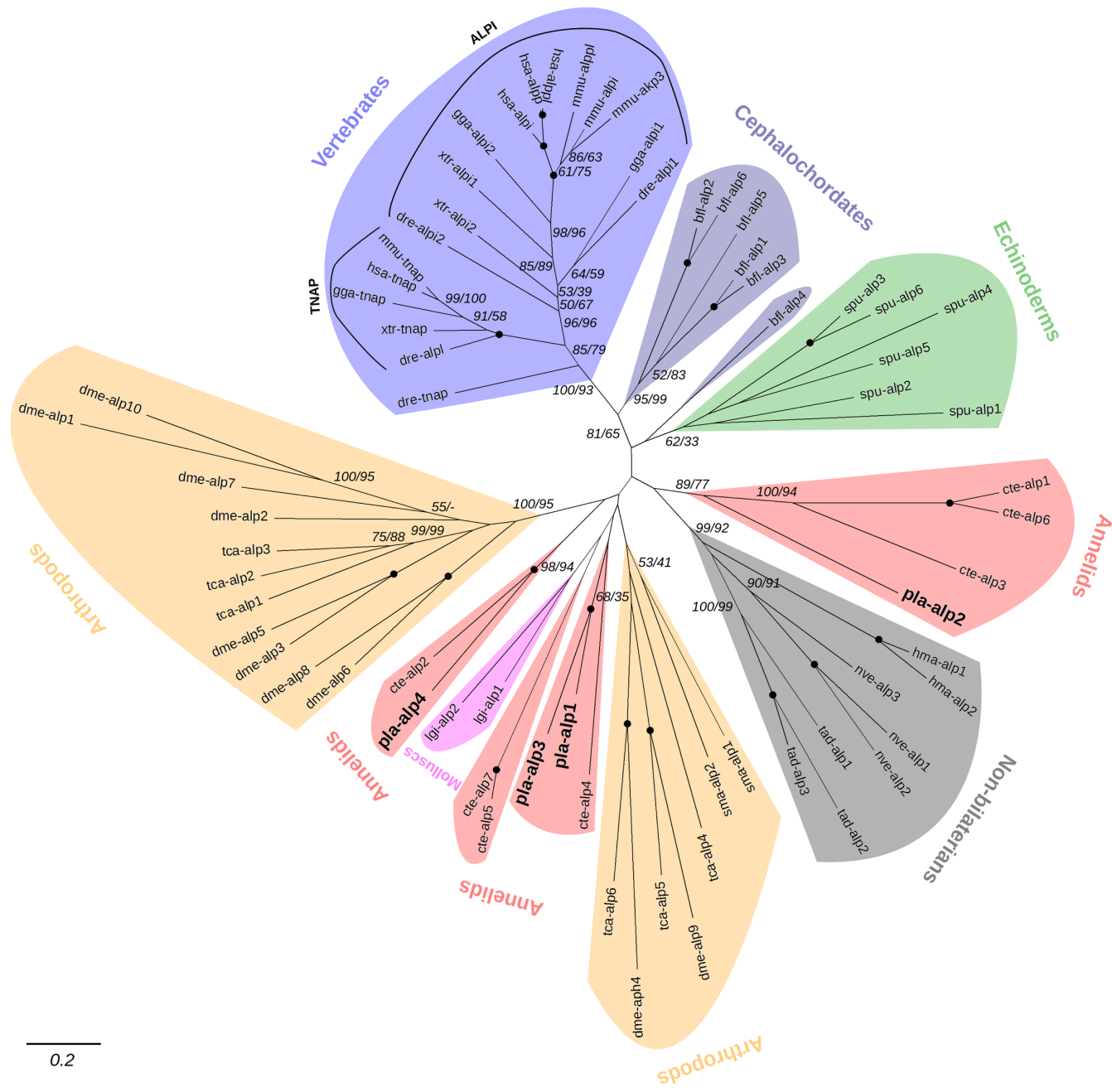

Fig. 6. Relationships of metazoan alkaline phosphatases. Unrooted maximum likelihood phylogenetic tree of 69 metazoan alkaline phosphatase protein sequences. (For alignment, accessions and sequence sources see the online supplementary material.) P. lamarckii sequences are highlighted by larger font and bolding. Vertebrate intestinal/placental (ALPI) and tissue-nonspecific (TNAP) alkaline phosphatase clades are labelled. Percentage bootstrap supports lat least $50 \%$ out of 500 replicates) in maximum likelihood and neighbourjoining trees are indicated next to branches. Branches that were not recovered in the neighbour-joining tree are indicated with a dash. Nodes with $100 \%$ support in both analyses are marked with a circle. Species abbreviations are: bfl, Branchiostoma floridae; cte, Capitella teleta; dme, Drosophila melanogaster; dre, Danio rerio; gga, Gallus gallus; hma, Hydra magnipapillata; hsa, Homo sapiens; Igi, Lottia gigantea; mmu, Mus musculus; nve, Nematostella vectensis; pla, Pomatoceros lamarckii; sma, Strigamia maritima; spu, Strongylocentrotus purpuratus; tad, Trichoplax adhaerens; tca, Tribolium castaneum; $x t r$, Xenopus tropicalis. 
ALP domain (Fig. 5). This fragment may belong to a fifth alkaline phosphatase gene whose expression domain lies mostly outside the opercular filament.

Alkaline phosphatase activity in the opercular filament is sensitive to levamisole, an uncompetitive ALP inhibitor. The availability of inhibitors opens the door to functional studies; nevertheless, the broad toxicity of levamisole makes interpretation of in vivoexposure experiments difficult (personal observation). Other, less toxic and/ or more specific inhibitors may offer a better alternative for elucidating the role of alkaline phosphatases in opercular regeneration.

Consistent with previous studies, phylogenetic analysis of the alkaline phosphatase proteins of selected metazoans reveals widespread lineage-specific duplications. Vertebrate ALP nomenclature clearly cannot be applied outside of Vertebrata. In fact, our analysis confirms earlier conclusions (Yang et al., 2012) that independent duplications have occurred even within the vertebrates, although a more thorough discussion of vertebrate ALP evolution is outside the scope of this study. The deep nodes of the tree are poorly resolved, but strongly supported clades are seen within phyla. Notably, all four $P$. lamarckii sequences group with those of the other annelid in the analysis, Capitella teleta, although the affinity of pla-alp1 and pla-alp3 with their putative $C$. teleta orthologue is poorly supported. Some $C$. teleta sequences (cte-alp5 and cte-alp7) have no candidate orthologue in $P$. lamarckii; whether this is due to the poor resolution of the tree, gene loss in the Pomatoceros lineage, or merely the absence of $P$. lamarckii alkaline phosphatases not expressed in the opercular filament from our organ-specific transcriptomes remains to be seen.

Opercular regeneration in Pomatoceros lamarckii is characterised by extensive, dynamic and strongly regionalised alkaline phosphatase activity. Staining is present in broad regions of regenerating and mature peduncles, and changes in staining intensity or distribution are associated with significant morphological features such as the easy break point and the opercular rim. Notably, we did not observe significant activity in the epidermis, including the epidermis of the calcifying opercular plate, indicating that alkaline phosphatases are not tightly associated with mineral deposition in the operculum. The broad distribution of staining in the peduncle and its persistence in mature specimens suggests that alkaline phosphatases have roles other than dedifferentiation or stem cell activity. The opercular filament expresses at least four distinct alkaline phosphatases, with phylogenetic analysis implying the orthology of these proteins to other annelid sequences and widespread lineage-specific duplications in the ALP family throughout the Metazoa.

\section{Materials and Methods}

\section{Animals}

Rocks settled by adult $P$. lamarckii were collected from the intertidal rock pools off East Sands beach, St Andrews, Scotland. Whole rocks were kept in a flow-through seawater aquarium at ambient temperature until needed. To obtain the animals, the posterior end of the tube was broken open with blunt forceps and the worm pushed out from the other end. Detubed animals were kept in $9 \mathrm{~cm}$ plastic Petri dishes containing 25-30 ml filtered seawater (FSW). Experimental animals were maintained in an air-conditioned room at $15-16^{\circ} \mathrm{C}$ and constant darkness, except for handling. All opercular amputations were carried out with a scalpel at the easy break point.

\section{Alkaline phosphatase assay}

ALP activity in the opercular filament was assayed with the chromogenic substrate NBT/BCIP (Roche). The anterior ends of worms were removed with a scalpel and fixed for half an hour at room temperature or overnight at $4{ }^{\circ} \mathrm{C}$ in phosphate buffered saline (PBS) containing $4 \%$ paraformaldehyde (PFA). The fixative was removed with three PBS washes, and the opercular filament was dissected from the specimens and placed in PBS with $0.1 \%$ Tween-20 (PBT). Some specimens were cut into portions with a razorblade. For early regenerates (1-2 dpo), a transverse cut was made near the base of the swelling indicating the presumptive cup. Specimens in mid-regeneration (3-4 dpo) were cut into a proximal and distal peduncle, and left and right cup halves. In older regenerates and mature specimens, the peduncle was cut into thirds rather than halves.

Dissected specimens were washed twice with AP buffer ( $0.1 \mathrm{M}$ Tris, 0.1 $\mathrm{M} \mathrm{NaCl}, 0.05 \mathrm{M} \mathrm{MgCl}_{2}$ and $0.1 \%$ Tween-20) before incubation in staining buffer (AP buffer with $7.5 \mu \mathrm{l} / \mathrm{ml}$ NBT/BCIP) at room temperature. Staining took approximately 30 to 80 minutes. Stained specimens were washed three times in PBT, post-fixed and washed three times in PBT. Before washing into AP buffer, the whole opercular filaments shown in Fig. 1 (except Fig. $1 \mathrm{H}$ ) were also treated with a 1:100 dilution of Sigma molecular grade Proteinase $\mathrm{K}$ at $37^{\circ} \mathrm{C}$ for either 15 minutes (regenerates) or 30 minutes (unoperated specimens). Proteinase $\mathrm{K}$ treated specimens were washed three times in PBT and post-fixed in PFA at room temperature before being moved into AP buffer. Proteinase treatment does not appreciably affect the staining pattern, but it considerably reduces nonspecific dye precipitation in the cuticle, which is especially a problem with older regenerates and mature opercular filaments (Fig. 1H, Fig. 3 B-D).

Imaging of the general staining pattern was carried out in PBT using a Nikon Coolpix 4500 digital camera mounted on a dissecting microscope. For more detailed images (Fig. 2), specimens were dehydrated through an ethanol series, cleared in $60 \%$ glycerol and mounted in Vectashield mounting medium (Vector Laboratories). Imaging of these specimens was carried out using a QImaging Retiga 2000R camera mounted on a Leica microscope equipped with Nomarski optics, and the QCapture Suite (TM) version 2.9.13.

To test whether opercular alkaline phosphatases are sensitive to the ALP inhibitor levamisole, three sets of 6 dpo regenerates were fixed and stained as above (without Proteinase K), but either deionised water or levamisole at a concentration of $2 \mathrm{mM}$ or $10 \mathrm{mM}$ was added to the staining buffer ( $n$ $=7$ for each treatment). For comparability, all three samples were stained for the same length of time (approx. 50 minutes at room temperature).

\section{Alkaline phosphatase sequences in Pomatoceros and Metazoa}

We also explored the evolutionary context of opercular alkaline phosphatases using sequence data from $P$. lamarckii and other animals. One $P$. lamarckii alkaline phosphatase protein sequence was obtained from the larval transcriptome assembly of Kenny and Shimeld (2012) (contig 42380). This sequence was detected, and three more putative paralogues were manually assembled from our own RNA-seq datasets using iterative tBLASTn and BLASTn searches (Altschul et al., 1997) against raw paired-end reads of $100 \mathrm{bp}$. RNA-seq data were derived from unoperated, 2 dpo and 6 dpo opercular filaments. The three new P. lamarckii alkaline phosphatase sequences are provided in the supplementary information.

Alkaline phosphatase protein sequences from other metazoans were obtained from the NCBI databases and from publicly available genome databases (for a full list of sequences, database sources and corrections applied to published sequences see the supplementary material). Our aim was to examine the diversity of metazoan ALP sequences; therefore, taxa were chosen based on the existence of genomic data from a broad section of the animal kingdom. Searches of available sponge, ctenophore and choanoflagellate sequences yielded no results. For these taxa, the following databases were searched: NCBI nr and EST databases using BLASTp and tBLASTn searches restricted to Porifera, Ctenophora or Choanoflagellata (http://blast.ncbi.nlm.nih.gov); Amphimedon queenslandica 
genome assembly (Ensembl Metazoa, http://metazoa.ensembl.org/Amphimedon_queenslandica/Info/Index); Compagen datasets (http://www. compagen.org/blast.html) for the Oscarella carmela genome and ESTs and Mnemiopsis leidyi ESTs; Mnemiopsis leidyigenome and transcriptome (http://research.nhgri.nih.gov/mnemiopsis/).

Alkaline phosphatase domains based on cd00016 in the NCBI Conserved Domain Database (http://www.ncbi.nlm.nih.gov/Structure/cdd/cdd. shtml) were aligned from a total of 69 sequences from 16 species spanning most major metazoan clades. Initial alignment was done with MAFFT 6 (Katoh and Toh, 2010) accessed through Jalview 2.8 (Waterhouse et al., 2008). For refinement of poorly aligned regions, manual editing in MEGA 5 (Tamura et al., 2011) and realignment with MUSCLE (Edgar, 2004) implemented in MEGA 5 was used. The full alignment is shown in the supplementary material.

Neighbour-joining trees were generated using the default settings in MEGA with the exception that columns with missing data were deleted pairwise rather than completely, to maximise the information derived from a dataset including incomplete sequences. 500 bootstrap replicates were used to estimate tree robustness. For maximum likelihood analysis, model selection was done with Modelgenerator 0.85 (Keane et al., 2006); all three information criteria agreed on the same model (WAG $+I+\Gamma)$. ML tree inference was carried out in PhyML 3.0 (Guindon et al., 2010) accessed via the South of France bioinformatics platform (http://www.atgc-montpellier. $\mathrm{fr} / \mathrm{phyml} /$ ) with subtree pruning and regrafting as the search method and 500 bootstrap replicates.

\section{Acknowledgements}

The authors would like to thank Jonathan Henry for the opportunity to contribute this manuscript to this special issue, and the members of the Ferrier and Somorjai groups for discussions during the course of this work. $R S$ is supported by a Carnegie scholarship.

\section{References}

ALTSCHUL S F, MADDEN T L, SCHÄFFER A A, ZHANG J, ZHANG Z, MILLER W and LIPMAN D J (1997). Gapped BLAST and PSI-BLAST: a new generation of protein database search programs. Nucl Acids Res 25:3389-3402.

BUBEL A, STEPHENS R M, FENN R H, and FIETH P (1983). An electron microscope, X-ray diffraction and amino acid analysis study of the opercular filament cuticle, calcareous opercular plate and habitation tube of Pomatoceros lamarckil Quatrefages (Polychaeta: Serpulidae). Comp Biochem Physiol B 74: 837-850.

BUBELA, THORP CH, FENN R H, and LIVINGSTONE D (1985). Opercular regeneration in Pomatoceros lamarckii Quatrefages (Polychaeta: Serpulidae). Differentiation of the operculum and deposition of the calcareous opercular plate. J Zool1:49-94.

BUCHET R, MILLÁN J L, and MAGNE D (2013). Multisystemic functions of alkaline phosphatases. In Phosphatase Modulators (Ed. J L MILLÁN). Humana Press, New York, pp. 27-51.

CHOCKALINGAM S (1971). Studies on enzymes associated with calcification of the cuticle of the hermit crab Clibanarius olivaceous. Mar Biol 10: 169-182.

DOMART-COULON IJ, ELBERTD C, SCULLYEP, CALIMLIM PS, and OSTRANDER G K (2001). Aragonite crystallization in primary cell cultures of multicellular isolates from a hard coral, Pocillopora damicornis. Proc NatlAcad SciUSA 98: 11885-11890.

DONACHY J E, WATABE N, and SHOWMAN, R M (1990). Alkaline phosphatase and carbonic anhydrase activity associated with arm regeneration in the seastar Asterias forbesi. Mar Biol 105: 471-476.

EDGAR R C (2004). MUSCLE: multiple sequence alignment with high accuracy and high throughput. Nucl Acids Res 32: 1792-1797.

EGUCHI M (1995). Alkaline phosphatase isozymes in insects and comparison with mammalian enzyme. Comp Biochem Physiol B 111: 151-162.

GUINDON S, DUFAYARD, J-F, LEFORT V, ANISIMOVA, M, HORDIJK, W, and GASCUEL, O (2010). New algorithms and methods to estimate maximum-likelihood phylogenies: Assessing the performance of PhyML 3.0. Syst Biol 59: 307-321.

HANSON J (1949). Observations on the branchial crown of the Serpulidae (Annelida,
Polychaeta). Q J Microsc Sci s3-90: 221-233.

HASSE C, REBSCHER N, REIHER W, SOBJINSKI K, MOERSCHEL E, BECK L, TESSMAR-RAIBLE K, ARENDT D, and HASSEL M (2010). Three consecutive generations of nephridia occur during development of Platynereis dumerilii (Annelida, Polychaeta). Dev Dyn 239: 1967-1976.

HOHAGEN $J$ and JACKSON D J (2013). An ancient process in a modern mollusc: early development of the shell in Lymnaea stagnalis. BMC Dev Biol 13: 27.

ISAEVA V V, AKHMADIEVA A V, ALEKSANDROVA Y N, SHUKALYUK A I, and CHERNYSHEV A V (2011). Germinal granules in interstitial cells of the colonial hydroids Obelia longissima Pallas, 1766 and Ectopleura crocea Agassiz, 1862. Russ J Mar Biol 37: 303-310.

KATOH K and TOH H (2010). Parallelization of the MAFFT multiple sequence alignment program. Bioinformatics 26: 1899-1900.

KEANE T M, CREEVEY C J, PENTONY M M, NAUGHTON T J, and MCLNERNEY, $\mathrm{J} \mathrm{O}$ (2006). Assessment of methods for amino acid matrix selection and their use on empirical data shows that ad hoc assumptions for choice of matrix are not justified. BMC Evol Biol 6: 29.

KENNY N J and SHIMELD S M (2012). Additive multiple $k$-mer transcriptome of the keelworm Pomatoceros lamarckii (Annelida; Serpulidae) reveals annelid trochophore transcription factor cassette. Dev Genes Evol 222: 325-339.

KITAMURA K and SHIMIZU T (2000). Analyses of segment-specific expression of alkaline phosphatase activity in the mesoderm of the oligochaete annelid tubifex: Implications for specification of segmental identity. Dev Biol 219: 214-223.

MILLÁN J L (2013). The role of phosphatases in the initiation of skeletal mineralization. Calcif Tissue Int 93: 299-306.

MYOHARA M (2004). Differential tissue development during embryogenesis and regeneration in an annelid. Dev Dyn 231: 349-358.

O'CONNOR M D, KARDEL M D, IOSFINA I, YOUSSEF D, LU M, LI M M, VERCAUTEREN S, NAGYA, and EAVES C J (2008). Alkaline phosphatase-positive colony formation is a sensitive, specific, and quantitative indicator of undifferentiated human embryonic stem cells. Stem Cells 26: 1109-1116.

OSBORNE P J and MILLER A T (1963). Acid and alkaline phosphatase changes associated with feeding, starvation and regeneration in planarians. Biol Bull 124: 285-292.

PINONI S A, GOLDEMBERG A L, and LÓPEZ MAÑANES AA (2005). Alkaline phosphatase activities in muscle of the euryhaline crab Chasmagnathus granulatus: Response to environmental salinity. J Exp Mar Biol Ecol 326: 217-226.

RINKEVICH Y, VOSKOBOYNIK A, ROSNER A, RABINOWITZ C, PAZ G et al., (2013). Repeated, long-term cycling of putative stem cells between niches in a basal chordate. Dev Cell 24: 76-88.

SCHMIDT A J and WEARY M (1962). The distribution of alkaline phosphatase in the regenerating forelimb of the adult newt, Diemictylus viridescens (Triturus $v$.). $J$ Exp Zool 150: 69-81.

SHUKALYUK A, ISAEVA V, KIZILOVA E, and BAIBORODIN S (2005). Stem cells in the reproductive strategy of colonial rhizocephalan crustaceans (Crustacea: Cirripedia: Rhizocephala). Inver Rep Dev 48: 41-53.

SZABÓ R and FERRIER D E K (2014). Cell proliferation dynamics in regeneration of the operculum head appendage in the annelid Pomatoceros lamarckii. $J \mathrm{~J}$. Exp. Zool. (Mol. Dev. Evol.) 322B: 257-268.

SZABÓ R, CALDER AC and FERRIER DEK (2014). Biomineralisation during operculum regeneration in the polychaete Spirobranchus lamarcki. Mar Bio 161: 2621-2629.

TAMURA K, PETERSON D, PETERSON N, STECHER G, NEI M, and KUMAR S (2011). MEGA5: Molecular evolutionary genetics analysis using maximum likelihood, evolutionary distance, and maximum parsimony methods. Mol Biol Evol 28: 2731 -2739.

TIMMERMANS L P M (1969). Studies on shell formation in molluscs. Neth J Zool 19: 413-523.

WATERHOUSE A M, PROCTER J B, MARTIN D M A, CLAMP M, and BARTON G $\mathrm{J}$ (2009). Jalview Version 2-a multiple sequence alignment editor and analysis workbench. Bioinformatics 25: 1189-1191.

YANG Y, WANDLER A M, POSTLETHWAIT J H, and GUILLEMIN K (2012). Dynamic evolution of the LPS-detoxifying enzyme intestinal alkaline phosphatase in zebrafish and other vertebrates. Front Immuno/ 3: 314 


\section{Further Related Reading, published previously in the Int. J. Dev. Biol.}

Brachyury, Tbx2/3 and sall expression during embryogenesis of the indirectly developing polychaete Hydroides elegans Cesar Arenas-Mena

Int. J. Dev. Biol. (2013) 57: 73-83

Characterization of novel F-actin envelopes surrounding nuclei during cleavage of a polychaete worm.

S Jacobsohn

Int. J. Dev. Biol. (1999) 43: 19-26

Cytoskeletal mechanisms of ooplasmic segregation in annelid

\section{eggs.}

T Shimizu

Int. J. Dev. Biol. (1999) 43: 11-18

Metabolism of oocyte construction and the generation of histospecificity in the cleaving egg. Lessons from nereid annelids.

A Fischer, A W Dorresteijn and U Hoeger

Int. J. Dev. Biol. (1996) 40: 421-430

Metamorphosis and pattern formation in Hydractinia echinata, a colonial hydroid

M Walther, R Ulrich, M Kroiher and S Berking

Int. J. Dev. Biol. (1996) 40: 313-322

http://dx.doi.org/10.1387/ijdb.8735943

5 yr ISI Impact Factor $(2011)=2.959$
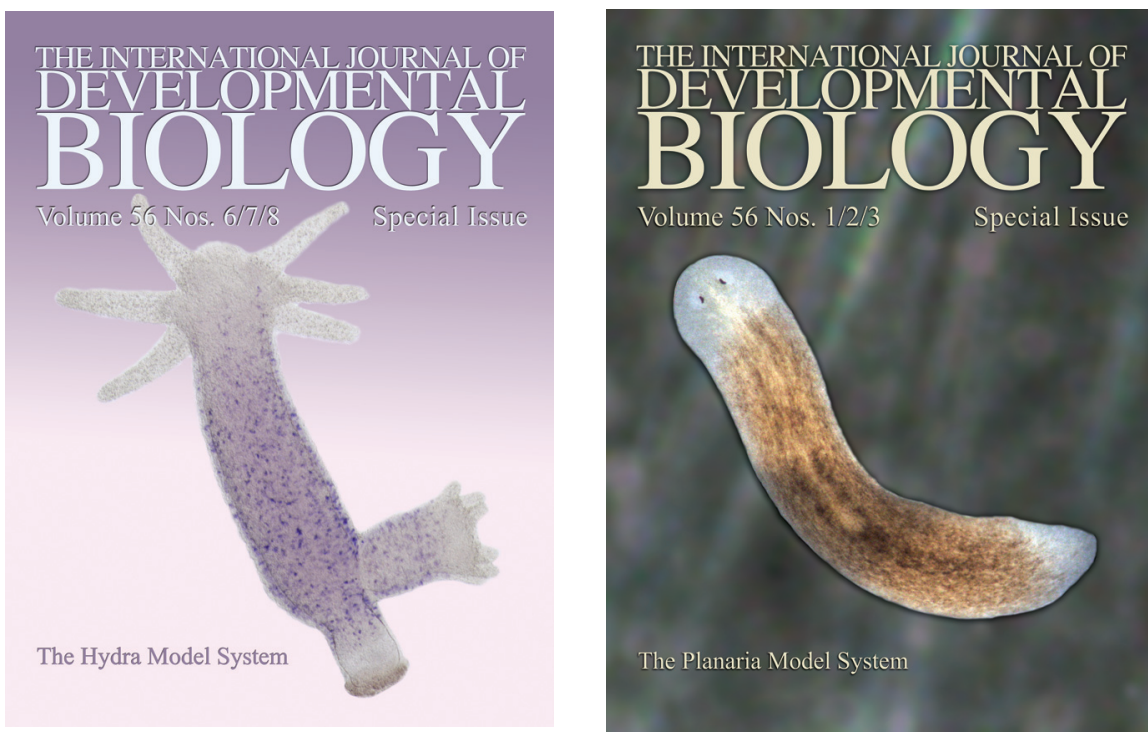
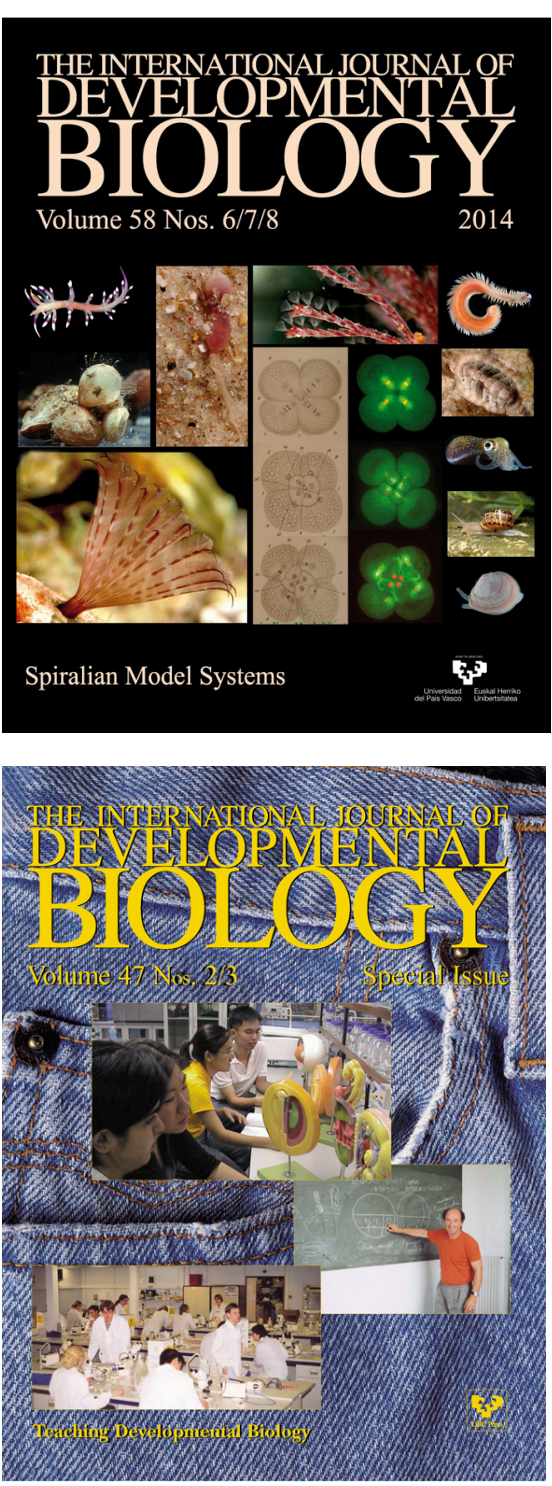